\title{
Heart Motion Abnormality Detection via an Information Measure and Bayesian Filtering
}

\author{
Kumaradevan Punithakumar ${ }^{1}$, Shuo Li $^{1}$, Ismail Ben Ayed ${ }^{1}$, Ian Ross ${ }^{2}$, \\ Ali Islam $^{3}$, and Jaron Chong ${ }^{4}$ \\ 1 GE Healthcare, London, ON, Canada \\ 2 London Health Science Center, London, ON, Canada \\ 3 St. Joseph's Health Care, London, ON, Canada \\ 4 University of Western Ontario, London, ON, Canada
}

\begin{abstract}
This study investigates heart wall motion abnormality detection with an information theoretic measure of heart motion based on the Shannon's differential entropy (SDE) and recursive Bayesian filtering. Heart wall motion is generally analyzed using functional images which are subject to noise and segmentation inaccuracies, and incorporation of prior knowledge is crucial in improving the accuracy. The Kalman filter, a well known recursive Bayesian filter, is used in this study to estimate the left ventricular (LV) cavity points given incomplete and noisy data, and given a dynamic model. However, due to similarities between the statistical information of normal and abnormal heart motions, detecting and classifying abnormality is a challenging problem which we proposed to investigate with a global measure based on the SDE. We further derive two other possible information theoretic abnormality detection criteria, one is based on Rényi entropy and the other on Fisher information. The proposed method analyzes wall motion quantitatively by constructing distributions of the normalized radial distance estimates of the LV cavity. Using $269 \times 20$ segmented LV cavities of short-axis magnetic resonance images obtained from 30 subjects, the experimental analysis demonstrates that the proposed SDE criterion can lead to significant improvement over other features that are prevalent in the literature related to the LV cavity, namely, mean radial displacement and mean radial velocity.
\end{abstract}

\section{Introduction}

Early detection of motion abnormality is the utmost importance in the diagnosis of coronary heart disease - the most common type of cardiovascular disease. Unfortunately, early detection by visual inspection is limited due to vast amount of information and uncertainty associated with heart motion. Computer-aided detection systems, which can analyze extensive amount of information associated with the heart motion, have attracted research attention in recent years [12]3. Computer-aided abnormality detection primarily consists of two components: preprocessing and classification. 
The preprocessing, centered around image segmentation, is in itself challenging due to the difficulties inherent to cardiac images [4. Additionally, the classification is also difficult because of similarities between the statistical information associated with normal and abnormal heart motion. Fig. 1]depicts typical examples of normal and abnormal heart motion, along with the corresponding distributions of motion measurements over time. The significant overlap between these distributions makes the classification problem difficult, and the use of distribution moments, for instance the mean [2], may not be sufficient to separate normal and abnormal motions. To tackle the classification problem, we propose an information theoretic measure of heart motion. In order to take full advantage of the information related to cardiac motion, we propose to use the Shannon's differential entropy (SDE) [5], which provides a global, theoretically grounded measure of distributions - rather than relying on elementary measurements or a fixed set of moments, the SDE measures a global distribution information and, as such, has more discriminative power in classifying distributions. The typical examples in Fig. 1]illustrate the potential of the SDE in the classification problem: the means of abnormal and normal motion distributions are very close, whereas, the SDEs are relatively different.

We further derive two other possible information theoretic abnormality detection criteria, one is based on Rényi entropy and the other on Fisher information [5]. Although widely used in physics [6], computer vision [78, communications 9], and many other fields, the application of information theoretic concepts is still in its early stage in medical image analysis. Few notable exceptions include

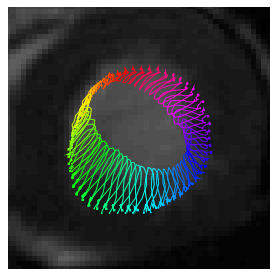

(a) Normal

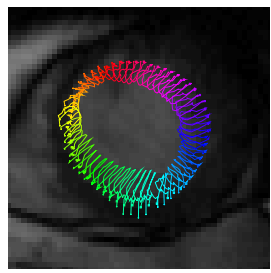

(b) Abnormal

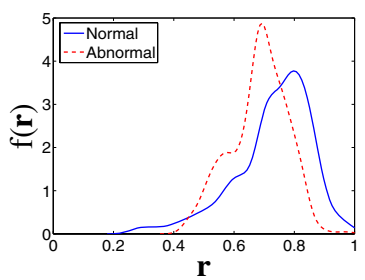

(c) Radial distance

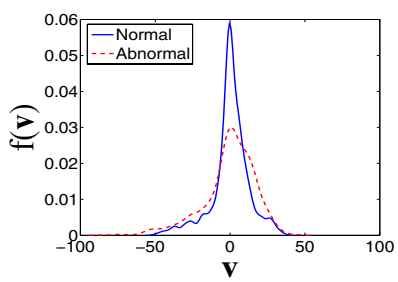

(d) Radial velocity
Radial distance statistics:

$\operatorname{Mean}($ normal $)=0.73$

Mean (abnormal) $=0.68$

$\mathrm{SDE}($ normal $)=-0.69$

$\operatorname{SDE}($ abnormal $)=-0.94$

Radial velocity statistics:

$\operatorname{Mean}($ normal $)=0.0017$

Mean (abnormal $)=-0.0025$

$\operatorname{SDE}($ normal $)=3.8$

$\mathrm{SDE}($ abnormal $)=4.2$

Fig. 1. The potential of the SDE measure in detecting abnormal motion. (a) typical normal motion, (b) typical abnormal heart, (c) and (d) corresponding distributions of radial distances and radial velocities. A significant overlap exists between normal and abnormal motion distributions, and the corresponding first moments are approximately the same, whereas the SDEs are relatively different. 
using cross and joint entropy for image registration [1011], the Rényi entropy for measuring the heart rate Gaussianity [12, and the Shannon entropy for analyzing heart period variability [13.

To tackle image preprocessing, an overlap prior based left ventricle (LV) segmentation [4], which does not require a training, is used, and the segmentation results are subsequently processed with recursive Bayesian filtering. The latter, which provides a temporal smoothing of the dataset given a suitable model, is shown to be very effective when the data is less reliable. Specifically, a cyclic model is used to characterize the dynamics of sample points of the segmented LV cavity and the Kalman filter [14] is used for state estimation. The filter estimates are subsequently analyzed to build an information theoretic classifier of heart motion.

Using 269 image sequences, each consisting of 20 segmented LV cavities of short-axis magnetic resonance functional images, obtained from 30 subjects, the experimental analysis demonstrates that the proposed information theoretic measure of heart motion can lead to significant improvement over other features that are prevalent in the literature related to the LV cavity, namely, the mean radial displacement and mean radial velocity [2]. Furthermore, an analysis based on Bhattacharyya distance (cf. plots in Fig. 3), which measures the separability of classes in classification problems, show that the SDE yields better classification ability amidst the stochastic nature of the cardiac motion and image segmentation inaccuracies.

\section{The Recursive Bayesian Filtering}

The analysis is performed by sampling a set of points along the segmented LV cavity. We assume a cyclic state-space model for the dynamics of sample points that characterize the temporal evolution of the points for a periodic heart motion. Let $\mathbf{x}_{k}^{i}=\left[\begin{array}{llllll}\bar{x}_{k}^{i} & x_{k}^{i} & \dot{x}_{k}^{i} & \bar{y}_{k}^{i} & y_{k}^{i} & \dot{y}_{k}^{i}\end{array}\right]^{T}$ be the state vector, consisting of mean position $\left(\bar{x}_{k}^{i}, \bar{y}_{k}^{i}\right)$, current position $\left(x_{k}^{i}, y_{k}^{i}\right)$ and velocity $\left(\dot{x}_{k}^{i}, \dot{y}_{k}^{i}\right)$ of sample point $i$, respectively, in $x$ and $y$ coordinate directions at time step $k$ (for $k=1, \ldots, K$ ). The state transition equation for cyclic motion is given by

$$
\mathbf{x}_{k}^{i}=F_{k-1} \mathbf{x}_{k-1}^{i}+v_{k-1}^{i} \quad \text { for } i=1, \ldots, N
$$

where $F_{k}=\left[\begin{array}{ll}A_{k} & \mathbf{0}_{3 \times 3} \\ \mathbf{0}_{3 \times 3} & A_{k}\end{array}\right], A_{k}=\left[\begin{array}{ccc}1 & 0 & 0 \\ 1-\cos (\omega T) & \cos (\omega T) & \frac{1}{\omega} \sin (\omega T) \\ \omega \sin (\omega T) & -\omega \sin (\omega T) & \cos (\omega T)\end{array}\right],\left\{v_{k-1}^{i}\right\}$ is a zero-mean Gaussian noise sequence with covariance $Q_{k}, \omega$ the reciprocal of period of heart cycle, and $T$ the interval between two subsequent image frames. The additive noise in the dynamic model is an approximation, and is included to accommodate significant differences between the modeling and real motion of the LV cavity points.

The measurement equation is given by

$$
\mathbf{z}_{k}^{i}=H_{k} \mathbf{x}_{k}^{i}+w_{k}^{i} \quad \text { for } i=1, \ldots, N
$$


where $H_{k}=\left[\begin{array}{ll}B_{k} & \mathbf{0}_{1 \times 3} \\ \mathbf{0}_{1 \times 3} & B_{k}\end{array}\right], B_{k}=\left[\begin{array}{lll}0 & 1 & 0\end{array}\right]$ and $\left\{w_{k}^{i}\right\}$ is a zero-mean Gaussian noise sequence with covariance $R_{k}$. The measurements are obtained by sampling the segmented LV cavities. The measurement equation indicates the fact that only the current position of a sample point is measured.

The Kalman filter, which yields an optimal estimate for linear/Gaussian systems, is applied for state estimation. In some very rare cases, the segmentation results of the LV deviate significantly, and such inconsistencies are detected by gating the center of the segmented LV. The segmentation results are ignored in such cases, and the sample points were only predicted using the dynamic model, i.e., they were not updated by filter.

In order to find the sequence of corresponding points over time, the symmetric nearest neighbor correspondences [15] is applied by sampling a set of equallyspaced points along the LV boundary. The construction of a sequence of points is essential to analyze wall motion regionally. Using spline interpolation, $N_{s}$ points were sampled along the LV cavity in each frame, and $N$ points were chosen as measurements to the filter. A kernel density estimation based on normal kernel function is applied to obtain the probability density. The radial distance for each dataset is normalized with respect to maximum value, which allows analyzing different long-axis segments, namely, apical, mid and basal, without additional processing.

\section{The SDE of Normalized Radial Distance}

We define the following normalized radial distance $r_{k}^{i}$ by

$$
r_{k}^{i}=\frac{\sqrt{\left(\hat{x}_{k}^{i}-\frac{1}{N} \sum_{i} \hat{x}_{k}^{i}\right)^{2}+\left(\hat{y}_{k}^{i}-\frac{1}{N} \sum_{i} \hat{y}_{k}^{i}\right)^{2}}}{\max _{i} \sqrt{\left(\hat{x}_{k}^{i}-\frac{1}{N} \sum_{i} \hat{x}_{k}^{i}\right)^{2}+\left(\hat{y}_{k}^{i}-\frac{1}{N} \sum_{i} \hat{y}_{k}^{i}\right)^{2}}}
$$

where $\hat{x}_{k}^{i}$ and $\hat{y}_{k}^{i}$ are the estimates of $x_{k}^{i}$ and $y_{k}^{i}$, respectively. Let $\mathbf{r} \in \mathbb{R}$ be a random variable. The kernel density estimate of the normalized radial distance is given by

$$
f(\mathbf{r})=\frac{\sum_{i, k} \mathcal{K}\left(r_{k}^{i}-\mathbf{r}\right)}{N K}
$$

where $\mathcal{K}(y)=\frac{1}{\sqrt{2 \pi \sigma^{2}}} \exp \left(-\frac{y^{2}}{2 \sigma^{2}}\right)$ is the Gaussian kernel. In this study, we derive the SDE measure of heart motion as follows

$$
S_{f}=-\int_{\mathbf{r} \in \mathbb{R}} \frac{\sum_{i, k} \mathcal{K}\left(r_{k}^{i}-\mathbf{r}\right)}{N K}\left(\ln \sum_{i, k} \mathcal{K}\left(r_{k}^{i}-\mathbf{r}\right)-\ln N K\right) d \mathbf{r}
$$

We further derive two other information theoretic criteria to measure the global information associated with heart motion, one is based on the Rényi entropy

$$
R_{f}^{\alpha}=\frac{1}{1-\alpha} \ln \int_{\mathbf{r} \in \mathbb{R}}\left(\frac{\sum_{i, k} \mathcal{K}\left(r_{k}^{i}-\mathbf{r}\right)}{N K}\right)^{\alpha} d \mathbf{r} \quad \text { for } 0<\alpha<\infty, \alpha \neq 1
$$


and the other on Fisher information

$$
I_{f}=4 \int_{\mathbf{r} \in \mathbb{R}}|\nabla g(\mathbf{r})|^{2} d \mathbf{r},
$$

where

$$
g(\mathbf{r})=\sqrt{\frac{\sum_{i, k} \mathcal{K}\left(r_{k}^{i}-\mathbf{r}\right)}{N K}} .
$$

\section{Experiments}

The data contain 269 short-axis image sequences, each consisting of 20 functional 2D-images acquired from 20 normal and 10 abnormal hearts. The data were acquired on $1.5 \mathrm{~T}$ MRI scanners with fast imaging employing steady state acquisition (FIESTA) image sequence mode. The Kalman filter positions and velocities were initialized using two-point initialization [14, and mean positions were initialized using all the measurements in the sequence.

The experiments compare the proposed information theoretic measure based on the SDE with other classifier elements, namely, the mean radial displacement and mean systolic radial velocity, as well as other information measures, namely, Rényi entropy $(\alpha=2)$ and Fisher information. Radial velocity computations are based on the systolic phase of cardiac cycle. The results were compared with ground truth classification of the cine MRI sequences by experienced medical professionals. A heart is considered to be abnormal in an image sequence if any of its segments [16] is abnormal.

We used two criteria to measure the performance of each classifier element, namely, classification accuracy via leave-one-subject-out method 1 and the receiver operating characteristic (ROC) curves with corresponding area under the curves (AUCs). Furthermore, we used the Bhattacharyya measure to assess the discriminative power of each classifier elements. Table 1 summarizes the results.

The ROC and AUC. The ROC curves for classifier elements is shown in Fig. 2. The more inclined the curve towards the upper left corner, the better the classifier's ability to discriminate between abnormal and normal hearts. The figure shows that the proposed SDE has superior classifying ability than other classifier elements. The AUCs that correspond to the ROC curves in Fig. 2 are reported in Table 1. The AUC represents the average of the classifier sensitivity over false-positive resulting from considering different threshold values, and gives an overall summary of the classification accuracy. The SDE yielded the highest AUC and, therefore, has the best performance.

The Bhattacharyya measure. We used the Bhattacharyya distance metric to evaluate the overlap between the distributions of classifier elements over normal and abnormal motions. The Bhattacharyya metric [17] is given by

$$
\mathcal{B}=\sqrt{1-\sum_{y \in \mathbb{R}} \sqrt{f_{N}(y) f_{A}(y)}}
$$

\footnotetext{
${ }^{1}$ Each subject is classified given the information learned from other subjects.
} 
where $f_{N}$ and $f_{A}$ are the distributions over, respectively, normal and abnormal hearts. The higher $\mathcal{B}$, the lesser the overlap (Refer to Fig. 3 for an illustration) and, therefore, the better the discriminative ability of the classifier. The SDE yielded the highest $\mathcal{B}$ as reported in Table 1 and, therefore, the best discriminative ability. This is consistent with the previous findings based on ROC/AUC evaluations.

Classification accuracy. Evaluating the percentage of correctly classified hearts using leaving-one-subject-out method, the proposed SDE yielded $90.5 \%$ true positive (TP) and $78.6 \%$ true negative (TN), i.e., $90.5 \%$ of abnormal hearts and $78.6 \%$ of normal hearts were classified correctly, which is the best overall performance among the reported classifier elements in Table 1 .

Table 1. The area under the curve corresponding to Fig. 2. Bhattacharyya distance metric of normal/abnormal distributions given in Fig. 3. and the percentage of classification accuracy using leaving-one-subject-out method for classifier elements

\begin{tabular}{|c|c|c|c|c|}
\hline Classifier element & $\mathrm{AUC}(\%)$ & $\begin{array}{l}\text { Bhattacharyya } \\
\text { distance } \\
\text { metric }(\mathcal{B})\end{array}$ & $\begin{array}{l}\text { Classification } \\
\text { Abnormal (\%) }\end{array}$ & $\begin{array}{l}\text { n accuracy } \\
\text { Normal }(\%)\end{array}$ \\
\hline Mean systolic velocity & 70.8 & 0.32 & 79.4 & 54.9 \\
\hline Mean radial displacement & 87.3 & 0.53 & 76.2 & 70.9 \\
\hline Fisher information & 89.3 & 0.59 & 84.1 & 85.0 \\
\hline Rényi entropy & 90.8 & 0.60 & 87.3 & 84.5 \\
\hline Shannon's differential entropy & 90.9 & 0.62 & 90.5 & 78.6 \\
\hline
\end{tabular}

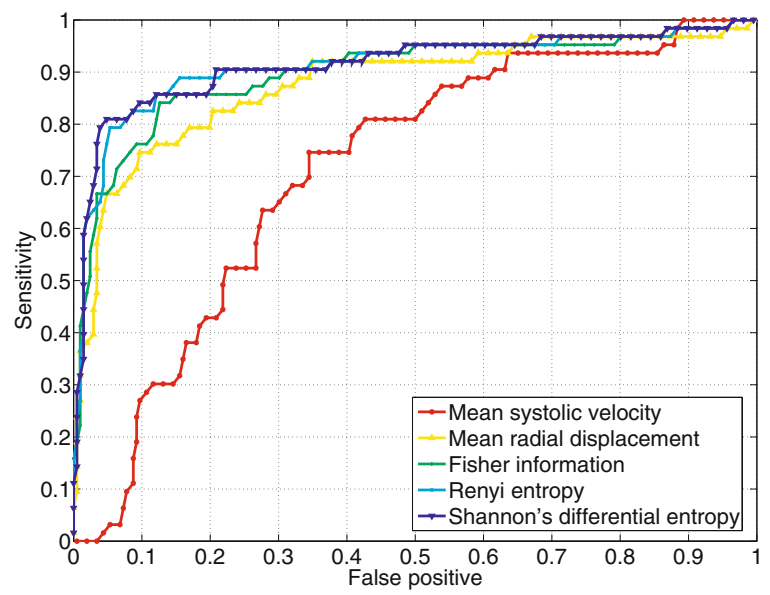

Fig. 2. Receiver operating characteristics of classifier elements. The closer the curve to the left hand top corner, the better the classification performance. The proposed information theoretic measure based on the SDE outperforms other classifier elements. 

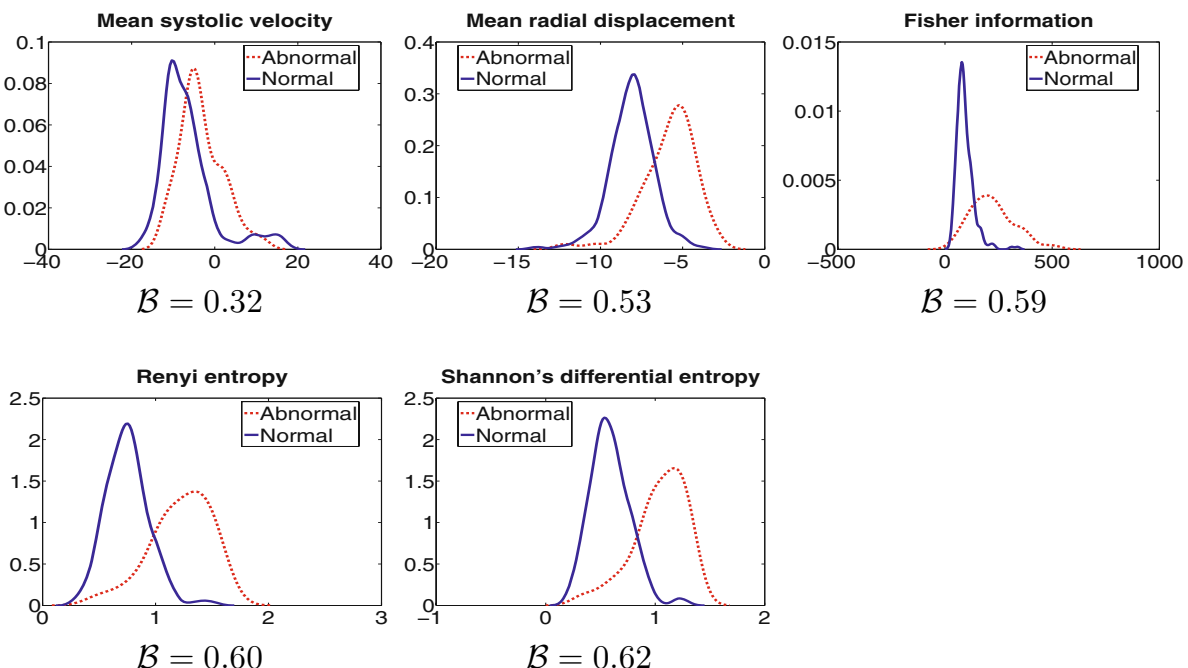

Fig. 3. Distribution of normal and abnormal hearts categorized using classifier elements. The Bhattacharyya distance metric show that information theoretic measure based on the SDE has better discriminative ability over other classifier elements.

\section{Conclusions}

This study investigates heart wall motion abnormality detection, which primarily consists of two components: preprocessing and classification. In preprocessing, an overlap prior based segmentation is used to generate left ventricular (LV) contours and the results are subsequently processed using Kalman filter, given a cyclic dynamic model. More importantly, we propose an information theoretic measure based on the Shannon's differential entropy (SDE) for classification. The proposed method analyzes wall motion quantitatively by constructing distributions of the radial distance estimates of the LV cavity. We further derive two other possible information theoretic abnormality detection criteria, one is based on Rényi entropy and the other on Fisher information. The experimental analysis is performed using $269 \times 20$ short-axis magnetic resonance images obtained from 30 subjects. The results, based on receiver operating characteristics, area under the curves, Bhattacharyya distance metrics and leave-one-subject-out cross validation, show that the proposed SDE criterion can lead to significant improvement over other prevalent classifier elements.

\section{References}

1. Qian, Z., Liu, Q., Metaxas, D.N., Axel, L.: Identifying Regional Cardiac Abnormalities from Myocardial Strains Using Spatio-temporal Tensor Analysis. In: Metaxas, D., Axel, L., Fichtinger, G., Székely, G. (eds.) MICCAI 2008, Part I. LNCS, vol. 5241, pp. 789-797. Springer, Heidelberg (2008) 
2. Qazi, M., Fung, G., Krishnan, S., Bi, J., Bharat Rao, R., Katz, A.: Automated heart abnormality detection using sparse linear classifiers. IEEE Engineering in Medicine and Biology Magazine 26(2), 56-63 (2007)

3. Mansor, S., Noble, J.: Local wall motion classification of stress echocardiography using a Hidden Markov Model approach. In: 5th IEEE International Symposium on Biomedical Imaging: From Nano to Macro., pp. 1295-1298 (2008)

4. Ben Ayed, I., Lu, Y., Li, S., Ross, I.: Left Ventricle Tracking Using Overlap Priors. In: Metaxas, D., Axel, L., Fichtinger, G., Székely, G. (eds.) MICCAI 2008, Part I. LNCS, vol. 5241, pp. 1025-1033. Springer, Heidelberg (2008)

5. Cover, T.M., Thomas, J.A.: Elements of Information Theory. Wiley-Interscience, New York (1991)

6. Frieden, B.R.: Physics from Fisher Information: a Unification. Cambridge University Press, Cambridge (1998)

7. Ben Ayed, I., Li, S., Ross, I.: Tracking distributions with an overlap prior. In: IEEE Conference on Computer Vision and Pattern Recognition, pp. 1-7 (2008)

8. Kim, J., Fisher III, J.W., Cetin, A., Yezzi, A., Cetin, M., Willsky, A.: A nonparametric statistical method for image segmentation using information theory and curve evolution. IEEE Transactions on Image Processing 14(10), 1486-1502 (2005)

9. Biglieri, E., Proakis, J., Shamai, S.: Fading channels: information-theoretic and communications aspects. IEEE Transactions on Information Theory 44(6), 2619 2692 (1998)

10. Studholme, C., Hill, D.L.G., Hawkes, D.J.: An overlap invariant entropy measure of 3D medical image alignment. Pattern Recognition 32(1), 71-86 (1999)

11. Zhu, Y.M.: Volume image registration by cross-entropy optimization. IEEE Transactions of Medical Imaging 21, 174-180 (2002)

12. Lake, D.E.: Renyi entropy measures of heart rate Gaussianity. IEEE Transactions on Biomedical Engineering 53(1), 21-27 (2006)

13. Porta, A., Guzzetti, S., Montano, N., Furlan, R., Pagani, M., Malliani, A., Cerutti, S.: Entropy, entropy rate, and pattern classification as tools to typify complexity in short heart period variability series. IEEE Transactions on Biomedical Engineering 48(11), 1282-1291 (2001)

14. Bar-Shalom, Y., Li, X.R., Kirubarajan, T.: Estimation with Applications to Tracking and Navigation. Wiley-Interscience, New York (2001)

15. Papademetris, X., Sinusas, A., Dione, D., Constable, R., Duncan, J.: Estimation of 3-D left ventricular deformation from medical images using biomechanical models. IEEE Transactions on Medical Imaging 21(7), 786-800 (2002)

16. Cerqueira, M.D., Weissman, N.J., Dilsizian, V., Jacobs, A.K., Kaul, S., Laskey, W.K., Pennell, D.J., Rumberger, J.A., Ryan, T., Verani, M.S.: Standardized Myocardial Segmentation and Nomenclature for Tomographic Imaging of the Heart: A Statement for Healthcare Professionals From the Cardiac Imaging Committee of the Council on Clinical Cardiology of the American Heart Association Circulation. 105, 539-542 (2002)

17. Comaniciu, D., Ramesh, V., Meer, P.: Kernel-based object tracking. IEEE Transactions on Pattern Analysis and Machine Intelligence 25(5), 564-577 (2003) 